\title{
Poly-harmonic Signal Characterization Method and ADC Characterization Using Josephson Converter and Linear Regression Analysis
}

\author{
Sergey V. Sherstobitov ${ }^{1}$, Mariia V.Karpova ${ }^{1}$ \\ ${ }^{1}$ All-Russian Scientific Research Institute of Physicotechnical and Radio Engineering Measurements \\ (VNIIFTRI), Mendeleevo, Moscow region, Russia, 141570 \\ s_sherstobitov@rambler.ru; Lab-610@vniiftri.ru
}

\begin{abstract}
Summary:
The features and limiting accuracy characteristics of the method for measuring the characteristics of poly-harmonic signals and the main characteristics of the ADC such us effective number of bits, signal-to-noise ratio, harmonic distortion, dynamic range are considered, depending on the number of ADC bits using a Josephson converter and linear regression analysis method.
\end{abstract}

Keywords: ADC, DAC, Josephson voltage standard, linear regression method, harmonic test signal.

\section{Introduction}

ADCs are widely used in modern measuring instruments. The conversion accuracy and, accordingly, the measurement accuracy strongly depends on their characteristics. In many methods, to determine the characteristics of the ADC, input voltage signals of a constant or step type are used. However, often such signals are not included in the class of ADC working signals, for example, due to the presence of an input filter that cuts off the DC component of the signal. Most ADCs work with harmonic sinusoidal signals of alternating voltage, in which the useful information is in the values of amplitudes, phases of fundamental tone and harmonic components. Traditionally TRUE RMS thermoelectric converters have been used at the top level of AC voltage metrology, but their usage is limited by sinusoidal signals with low harmonic components. In the case of analog instruments, input filters are used to measure signal harmonics contents. In the case of digital processing, FFT, DFT algorithms are often used, which have their own limitations on application and limit the measurement error. The construction of reference generators of the test signals based on Josephson arbitrary waveform synthesizer and the development of techniques for determining the characteristics of the ADC is an urgent task.

\section{Description of the Method}

Progress in the field of AC voltage measurements is associated with the development of $A C$ voltage standards based on the Josephson effect $[1,2]$. At the output of such a device, it is possible to obtain both a programmable DC voltage output signal with an exact value and almost zero non-linearity, and AC waveform signals with an exact value of the RMS value of a sinusoidal signal or an exact value of harmonic components [3]. Authors developed algorithms for determining the characteristics of harmonic signals and the characteristics of the DAC/ADC using the regression analysis method. The process of measuring of the ADC characteristics can be implemented in the following sequence: 1 . Submission to the ADC input of an exemplary analog test signal included in the class of permissible signals with specified parameter values. 2. Recording a sequence of digital readings obtained in real-time operating mode in a digital storage device. 3 . Recovery of signal parameter values by compute of the recorded sequence using software based on linear regression method. 4. Comparison of the obtained parameter values with the given ones and calculation of the values of the ADC characteristics as signal conversion errors. The software consists of two blocks - a computer Generator of digital signals and a processing unit for digital implementations. The Generator creates sequences of digital samples for digital-to-analog converters on Josephson binary array, and for other types of DACs. It is also used to generate digital test signals when characterizing another signal processing software. The Generator module generates a digital signal corresponding to the harmonic signal model of the following form: 


$$
A(t, N)=A_{0}+\sum_{l=1}^{M} A_{l} \sin \left(2 \pi l F_{0}+\Psi_{l}\right)+B(t, \sigma)
$$

where $A_{0}$ is the constant component of the signal $A(t, N) ; \mathrm{A}_{1}, 2 \pi I F_{0}$ and $\psi_{l}$-amplitude, angular frequency and phase of the I-th harmonic of the sinusoidal signal component $A(t, N) ; M$ is the number of harmonics of the fundamental tone of the sinusoidal component in the signal $A(t$, $N), B(t, \sigma)$ is a random function describing the additive "white" noise with zero mean and with standard deviation equal to $\sigma$.

For processing digital implementations, regression analysis method is used. The internal scale parameter of the implementation is the number of samples per period of the fundamental tone. With its known value, the task of reconstructing the parameters of a sinusoidal signal is formulated as a linear regression by the principle of least squares in the class of sums of trigonometric functions. Then, the algorithm calculates the amplitudes and phases of the fundamental tone and harmonics, the coefficient of nonlinear distortion, the effective number of bits of the $A D C$, the signal-to-noise ratio, dynamic range, etc. To obtain the uncertainty errors in determining the frequency and amplitude parameters, a statistical modeling process was used.

\section{Results}

Modeling and processing were carried out taking into account five harmonics with a harmonic coefficient equal to $0.01 \%$. The noise model is normal white noise.

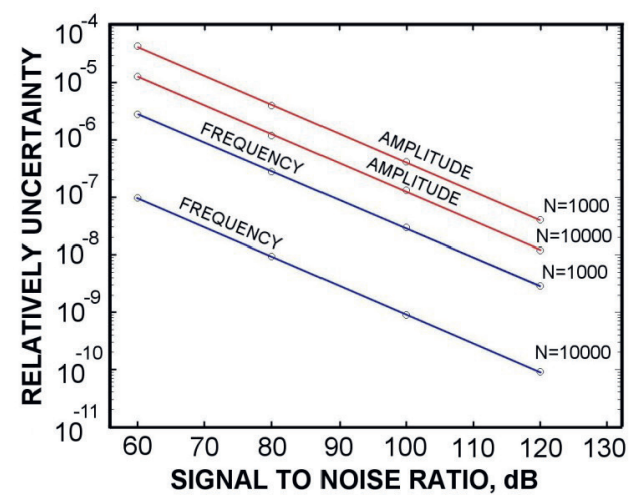

Fig. 1. The dependence of the relative errors in measurement the frequency and amplitude on the signal-to-noise ratio from $60 \mathrm{~dB}$ to $120 \mathrm{~dB}$ and number of samples $N=1000, N=10000$.

The number of sample parameter values for statistics was $500-10000$. The study of the graphs above shows that the error in the amplitude of the fundamental tone is inversely proportional to the signal-to-noise ratio and the number of counts in the signal implementation.
In practice, an increase in the implementation length (the number of processed samples) does not always lead to a decrease in the measurement error, since due to the instability of the real signal frequency, a phase error can accumulate, limiting the measurement accuracy. Implementation of the signal samples was loaded into the binary Josephson voltage converter and use to generate test signal for ADC. The output signal from the Josephson setup was fed to 24-bit ADC. Signals with a number of samples from 30 to 4000 for the period of the fundamental tone of the generated signal were used. Experiments were carried out to generate and measure the characteristics of signals with a different set of harmonic components, different coefficient of nonlinear distortion. In all cases, the method showed good accuracy in measuring both the amplitude of the fundamental tone and the harmonic components, which, makes it possible to estimate with high accuracy both the characteristics of the signal itself and the characteristics of the DAC/ADCs.

\section{Conclusion}

The results of statistical modeling using this method showed possible accuracy characteristics for measuring the parameters of the amplitude, phase, frequency of the fundamental tone of the signal and harmonics, which depend on the signal-to-noise ratio and the length of the processed implementation. The errors of the method when processing real signals will depend on the accuracy of the DAC/ADCs used, the accuracy and stability of the signal frequency and sampling frequency. Measurements with a Josephson setup, good results were obtained that were not achievable for other processing methods, such as FFT, DFT. The authors use these algorithms in the development of methods and instruments for measuring the characteristics of the ADCs and the characteristics of the AC Josephson setup to obtain uncertainty of amplitudes $1 \mathrm{ppm}$ and less.

\section{References}

[1] Hamilton C. A., Burroughs C. J., Kautz R. L.. Josephson D/A converter with fundamental accuracy, IEEE Trans. Instrum. Meas, 1995, vol. 44. no. 2. p. 223-225; doi: 10.1109/19.377816

[2] M. Sira, O. Kieler, R. Behr, A Novel Method for Calibration of ADC Using JAWS, EEE Trans. Instrum. Meas, 2019, vol. 68, no. 6, p. 2091 2099; doi:10.1109/TIM.2018.2888918

[3] S.P. Benz, F.L. Walls, PD Dresselhaus, C.J. Burroughs Low-Distortion Waveform Synthesis with Josephson Junction Arrays, IEICE Trans. Electron. 2002 vol. E85-C(No.3) p. 608-611 